Case Report

\title{
Scoliosis Correction Surgery for a Patient with Marfan Syndrome with Severe Scoliosis and Restricted Lung Ventilation
}

\author{
Liu Dongming ${ }^{1}$, Xiong Tingliang ${ }^{2}$, Zhang $\mathrm{Ziqi}^{3}$, Wang Xiaochen ${ }^{3}$, Zhang Guowei ${ }^{1}$, Yang Hua ${ }^{1}$, \\ Ji Zhisheng ${ }^{1, *}$, Lin Hongsheng ${ }^{1, *}$ \\ ${ }^{1}$ Department of Orthopedics, the First Affiliated Hospital of Jinan University, Guangzhou, China \\ ${ }^{2}$ The Second People's Hospital of Panyu Guangzhou, Guangzhou, China \\ ${ }^{3}$ School of Medicine, Jinan University, Guangzhou, China
}

Email address:

tzhishengji@jnu.edu.cn (Ji Zhisheng), tlinhsh@jnu.edu.cn (Lin Hongsheng)

${ }^{*}$ Corresponding author

\section{To cite this article:}

Liu Dongming, Xiong Tingliang, Zhang Ziqi, Wang Xiaochen, Zhang Guowei, Yang Hua, Ji Zhisheng, Lin Hongsheng. Scoliosis Correction Surgery for a Patient with Marfan Syndrome with Severe Scoliosis and Restricted Lung Ventilation. International Journal of Neurosurgery. Vol. 4, No. 2, 2020, pp. 63-67. doi: 10.11648/j.ijn.20200402.18

Received: December 1, 2020; Accepted: December 14, 2020; Published: December 25, 2020

\begin{abstract}
Objective: Marfan syndrome is a multi-system disease caused by genetic mutations that can cause damage to multiple systems in the body. With the development of the disease, the scoliosis of patients with Marfan syndrome will be further aggravated, which will eventually seriously affect the patient's cardiopulmonary function and lead to a serious decline in the patient's quality of life. The aim is to summarize the experience of spinal orthopedic surgery in a patient with Marfan syndrome and severe scoliosis with limited pulmonary ventilation. Method: In November 2019, a 13-year-old male with Marfan syndrome with severe scoliosis and restricted lung ventilation was admitted. The full-length positive and lateral X-rays of the spine before the operation showed that the spine was S-shaped scoliosis, double thoracic curve (thoracic curve + lumbar curve), upper vertebrae T4, lower vertebrae T11, thoracic T4-T11 structural curve, thoracic Cobb angle of bend is $42.4^{\circ}$, the lumbar bend is compensatory, Cobb angle of lumbar bend is $33.2^{\circ}$ The lung function indicates that the forced end-expiratory volume/forced vital capacity (FEV1/FVC) in the first second is 70\%. "Scoliosis correction + interlaminar bone graft fusion + posterior T4-T11 pedicle screw fixation" was performed on the patient under general anesthesia with tracheal intubation. Result: The full-length positive and lateral X-rays of the spine at 1 week, 1 month, 3 months, and 1 year after surgery showed that the internal fixation position was good, and the Cobb angle remained basically unchanged. There was basically no change in the Cobb angle of the thoracic curve $\left(10.2^{\circ}, 10.8^{\circ}\right.$, $10.9^{\circ}, 10.5^{\circ}$, respectively) and the Cobb angle of the lumbar curve $\left(13.1^{\circ}, 13.6^{\circ}, 13.8^{\circ}, 13.9\right.$, respectively) at each follow-up time point after surgery. The lung function at 1 year after surgery suggested that FEV1/FVC was $80 \%$. No sensorimotor abnormalities and other complications occurred during the follow-up. Conclusion: Scoliosis correction surgery is feasible and safe for patients with Marfan syndrome combined with severe scoliosis deformity and restricted lung ventilation. It can improve the patient's appearance, at the same time improve the patient's cardiopulmonary function, and solve the problem of the continued increase in the number of cobb angles of scoliosis. Surgery improves the quality of life of patients as a whole.
\end{abstract}

Keywords: Marfan Syndrome, Scoliosis, Spinal Deformity, Spinal Correction

\section{Introduction}

Marfan syndrome was first described by Antoine-Bernard Marfan, a doctor in Paris, France more than 100 years ago.
He reported that a 5-year-old girl had elongated fingers and other associated skeletal abnormalities [1]. The disease incidence of Marfan syndrome in England is 1/9802 [2]. Marfan syndrome is a systemic disease of connective tissue, 
caused by mutations in the FBN1 gene encoding fibrillin 1 or mutations in transforming growth factor-beta receptor 1 or 2 (TGFBR1or2) [3-7]. It mainly affects the cardiovascular system, skeletal system, and eyeball system. The skeletal system is usually manifested in the thoracic cage, vertebrae, and bone deformities of the hands and feet, such as pectus carinatum, scoliosis, overgrowth of long bones of the upper and lower limbs, etc. [8, 9]. When a patient with Marfan syndrome has severe scoliosis, it will seriously increase the patient's heart and lung burden [10]. At this time, it is particularly important to perform scoliosis correction surgery on the patient, which can not only improve the patient's appearance but also improve the patient's cardiopulmonary function in time and improve the patient's quality of life [11, 12]. So far, there are few reports of scoliosis correction in patients with Marfan syndrome. Our hospital admitted a patient with Marfan syndrome with severe scoliosis and restricted lung ventilation in November 2019. The diagnosis and treatment process is now reported as follows.

\section{Case Presentation}

\subsection{Case Data}

The patient, a male, 13 years old, was admitted to the hospital for "discovering bilateral shoulder height differences for 1 month and chest and back pain for 10 days". In the past 10 days, the patient developed chest and back pain and discomfort. When climbing 3 flights of stairs, he developed chest tightness, wheezing, and difficulty breathing. Physical examination: weight is $50 \mathrm{~kg}$, height is $180.6 \mathrm{~cm}$. The spine showed obvious scoliosis. The height of the shoulders was different on both sides. The skin creases on the right lower back were slightly raised, and the skin creases on the left lower back were sunken. When bending over, the shoulder blades on both sides show a "razorback" deformity with a high right and low left. The results of laboratory tests such as blood routine, urine routine, stool routine, liver and kidney function, and coagulation function were normal. The electrocardiogram showed sinus arrhythmia. Color Doppler ultrasound of the heart revealed prolapse of the anterior leaflet of the mitral valve, LVEF: $63 \%$, E/A: 67/42 = 1.60. The lung function indicates that the forced end-expiratory volume/forced vital capacity (FEV1/FVC) in the first second is $70 \%$. The full-length positive and lateral X-rays of the spine showed that the thoracic segment T4-T11 was a structural curve, the chest curve had a Cobb angle of $42.4^{\circ}$, the lumbar curve was a compensatory curve, and the lumbar curve had a Cobb angle of $33.2^{\circ}$. The upper vertebra is $\mathrm{T} 4$, the lower vertebra is $\mathrm{T} 11$, and Risser's sign is $4+$. The bending flexibility index is $57 \%$. The patient has had pectus carinatum orthopedic surgery in the past.

\subsection{Treatment Methods and Results}

\subsubsection{Preoperative Diagnosis and Surgical Protocol}

After the patient was admitted to the hospital, a series of routine examinations and condition evaluation were performed. The full-length positive and lateral X-rays of the spine showed that the spine showed an S-shaped scoliosis deformity, which was a double-curved structure of the thoracic curve and lumbar curve. The upper vertebra is T4, the lower vertebra is T11, the thoracic segment T4-T11 is a structural curve, the thoracic curve Cobb angle is $42.4^{\circ}$, the lumbar curve is a compensatory curve, and the lumbar curve Cobb angle is $33.2^{\circ}$. There is no obvious imbalance in the coronal plane of the spine, no obvious deformity in the sagittal plane of the spine, and the flexibility index of the bending position is $57 \%$. The top vertebra is at T8/9. CSVL is on the lateral side of the apical vertebrae, and the Lenke classification is $\mathrm{N}(+)$. lenke2C type. The diagnosis of Marfan syndrome with scoliosis $(\mathrm{N}(+)$. lenke2C type) and moderately restricted lung ventilation is clear. The surgical plan was general anesthesia for tracheal intubation and "scoliosis correction + interlaminar bone fusion + posterior T4-T11 pedicle screw fixation."

\subsubsection{Surgical Procedure}

The specific method: take the prone position, after the anesthesia is successful, indwell a urinary catheter, establish a deep venous channel, and apply electrophysiological monitoring and autologous blood transfusion during the operation. Routinely disinfect the surgical field, spread sterile towels, and paste sterile films. Take the longitudinal incision of the waist and back with the T4-T11 spinous process connection, the length is about $25 \mathrm{~cm}$. Cut the skin, subcutaneous connective tissue, and back fascia in turn, peel off the paravertebral muscles, and expose the T4-T11 bilateral facet joints. The $\mathrm{C}$-arm machine confirms that the T4 spinous process is correct. During the operation, the apex of the herringbone spine of the left facet joint of $\mathrm{T} 4$ was used as the needle entry point, followed by opening, reaming, sounding, and wiretapping. The probe was confirmed to enter the bony pedicle wall, and then the pedicle of appropriate length was screwed in the nail. In the same way, screw-in pedicle screws of appropriate length at the same level on the left side T4, T6, T8, T10, T11, and the opposite side. Intraoperative $\mathrm{C}$-arm fluoroscopy showed a good internal fixation position. Pre-bending the molding bar, pre-bend the longitudinal connecting rod according to the shape of the molded rod, insert the left nail tail, screw in the nut, rotate the longitudinal connecting rod, turn lumbar scoliosis into lumbar lordosis, and completely correct coronal scoliosis. Install the longitudinal connecting rod on the right side, screw in the nut, and also rotate until the coronal side bend is completely corrected and the nut is locked. Intraoperative $\mathrm{C}$-arm fluoroscopy showed that the internal fixation position was good, and the scoliosis was corrected. Wash the incision, bite off the T4-T11 supraspinous ligament, interspinous ligament, spinous process, and the obtained bone is used for bone grafting between the thoracic lamina. Then install the two horizontal connections. Exploration revealed that the internal fixation was in a good position and there was no obvious active bleeding. The incision was sutured layer by layer. The total operation time is 135 minutes, the intraoperative blood loss is about $500 \mathrm{~mL}$, and about $300 \mathrm{~mL}$ 
of autologous concentrated red blood cells are reinfused.

\subsubsection{Postoperative Treatment and Follow-up Results}

After the operation, ECG monitoring, blood oxygen monitoring, and related symptomatic treatment were given. One week after the operation, he got out of bed under the protection of a thoracolumbar brace. The full-length positive and lateral X-rays of the spine at 1 week, 1 month, 3 months, and 1 year after surgery showed that the internal fixation position was good, and the Cobb angle remained basically unchanged. There was basically no change in the Cobb angle
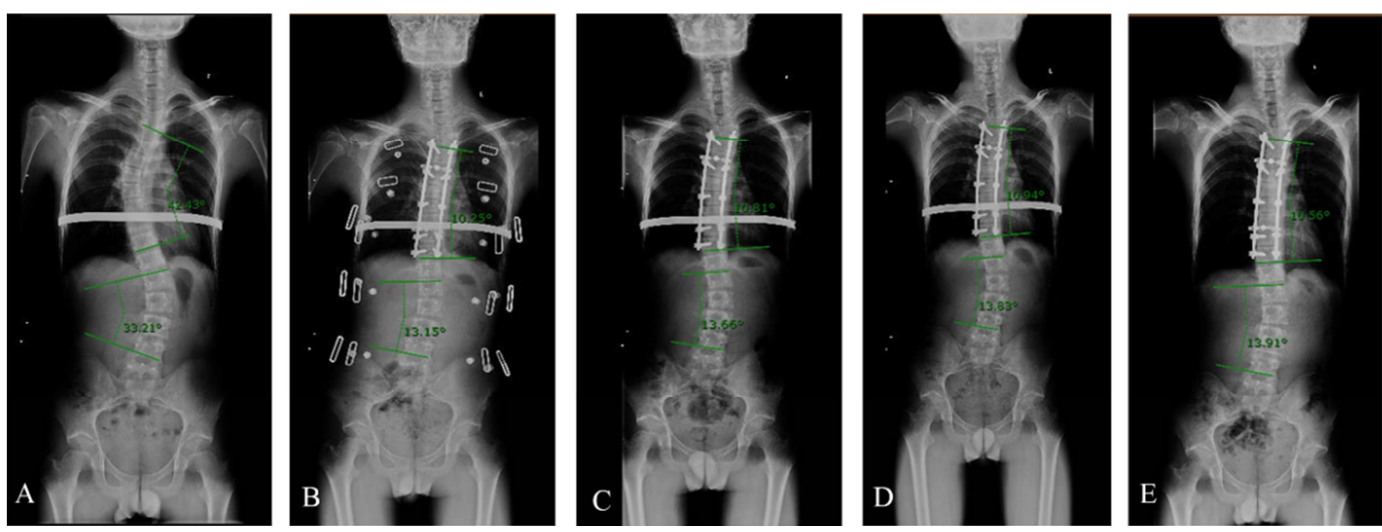

Figure 1. Imaging examinations of patients before and after surgery. A, is the full-length frontal X-ray of the spine before the operation, showing that the spine is deformed in scoliosis, showing a double-bend state of the thoracic and lumbar curve, in which the thoracic curve is a structural curve and the lumbar curve is a compensatory curve. $B, C, D$, and E are the full-length orthographic X-rays of the spine at 1 week, 1 month, 3 months, and 1 year after surgery, showing that the internal fixation position is good, and scoliosis has been corrected to a certain extent. And there was basically no change in the Cobb angle of chest bend and lumbar bend at each follow-up time.

\section{Discussion}

Marfan syndrome is a multi-system disease caused by genetic mutations, which can cause damage to multiple systems in the body [3-7]. The effects of the cardiovascular system are as follows: aortic expansion, with or without aortic regurgitation; mitral valve prolapse, with or without mitral regurgitation. Aortic dissection; aortic aneurysm and heart failure, etc., serious cardiovascular diseases can threaten the lives of patients $[9,10,13]$. The ocular system can be manifested as lens dislocation and ectropion, early and severe myopia, flat cornea, increased axial length of the sphere, iris dysplasia, and ciliary muscle hypoplasia, miosis, retinal detachment, and cataract or glaucoma [1, 9, 10, 13-15] The effects on the lungs may include: bullae, spontaneous pneumothorax, reduced lung capacity due to overgrowth of long bones or spinal deformities. The effect on the skin system can be manifested as skin wrinkles, inguinal hernias, and so on. The effects on the musculoskeletal system may include: pectus carinatum or pectus excavatum, excessive growth of long bones leads to slender limbs, scoliosis deformities of the spine, flat feet, etc. $[1,9,10,15,16]$. The impact of Marfan syndrome on the body is multi-system and multi-faceted. The disease will develop with age and cause serious damage to the body. Severe scoliosis in patients with Marfan syndrome can affect the development and function of the heart and lungs, resulting in a decline in exercise tolerance, which greatly reduces the patient's quality of life of the thoracic curve $\left(10.2^{\circ}, 10.8^{\circ}, 10.9^{\circ}, 10.5^{\circ}\right.$, respectively) and the Cobb angle of the lumbar curve $\left(13.1^{\circ}, 13.6^{\circ}, 13.8^{\circ}\right.$, 13.9 , respectively) at each follow-up time point after surgery. The height measured 1 week after the operation was $184 \mathrm{~cm}$, which was an increase of $3.4 \mathrm{~cm}$ compared with the preoperative height. Pulmonary function tests 1 year after surgery showed that the forced end-expiratory volume/forced vital capacity (FEV1/FVC) in the first second was $80 \%$. No sensorimotor abnormalities and other complications occurred during the follow-up. 
cardiopulmonary function of patients, and improve the quality of life of patients as a whole [17-20]. In this case, a 13-year-old patient with Marfan syndrome combined with severe scoliosis and moderately restricted pulmonary ventilation. Under general anesthesia for tracheal intubation, the operation of "scoliosis correction + interlaminar bone graft fusion + posterior T4-T11 pedicle screw fixation" was performed. Scoliosis correction surgery solves the unequal height of the patient's shoulders and increases the patient's height by $3.6 \mathrm{~cm}$. Through the follow-up after the operation, it was found that the cobb angle of the thoracic curve and the lumbar curve was basically unchanged. The lung function test of the patient 1 year after surgery showed that the forced end-expiratory volume/forced vital capacity (FEV1/FVC) in the first second increased from $70 \%$ before surgery to $80 \%$. Scoliosis correction surgery has achieved satisfactory results for this patient. While improving the patient's appearance, it also improves the patient's cardiopulmonary function and solves the problem of the continued increase in the number of cobb angles of scoliosis.

\section{Conclusion}

In this case report, scoliosis correction surgery solved the patient's unequal shoulder height and razorback problems, and at the same time increased the patient's height by $3.6 \mathrm{~cm}$. The overall appearance of the patient was improved. It can be seen from the postoperative follow-up that the lung function test of the patient 1 year after the operation showed that the forced end-expiratory volume/forced vital capacity (FEV1/FVC) in the first second increased from $70 \%$ to $80 \%$. The patient's cardiopulmonary function was also improved. The regular $\mathrm{X}$-ray examination after the operation showed that the Cobb angle of the thoracic curve and the cobb angle of the lumbar curve remained basically unchanged. In general, scoliosis correction surgery is feasible and safe for patients with Marfan syndrome combined with severe scoliosis deformity and restricted lung ventilation. It can improve the patient's appearance, at the same time improve the patient's cardiopulmonary function, and solve the problem of the continued increase in the number of cobb angles of scoliosis.

\section{Author Contributions}

Liu Dongming and Xiong Tingliang are co-first authors. They contributed equally to this work.

\section{Statement of Conflict of Interest}

All the authors do not have any possible conflicts of interest.

\section{References}

[1] Brindusa C: Marfan's syndrome: a daily struggle. The Lancet 2005, 366 (9501).
[2] Gray JR, Bridges AB, Faed MJ, Pringle T, Baines P, Dean J, Boxer M: Ascertainment and severity of Marfan syndrome in a Scottish population. J Med Genet 1994, 31 (1): 51-54.

[3] Loeys B, De Backer J, Van Acker P, Wettinck K, Pals G, Nuytinck L, Coucke P, De Paepe A: Comprehensive molecular screening of the FBN1 gene favors locus homogeneity of classical Marfan syndrome. Hum Mutat 2004, 24 (2): 140-146.

[4] Loeys B, Nuytinck L, Delvaux I, De Bie S, De Paepe A: Genotype and phenotype analysis of 171 patients referred for molecular study of the fibrillin-1 gene FBN1 because of suspected Marfan syndrome. Arch Intern Med 2001, 161 (20): 2447-2454.

[5] Mizuguchi T, Collod-Beroud G, Akiyama T, Abifadel M, Harada N, Morisaki T, Allard D, Varret M, Claustres M, Morisaki $\mathrm{H}$ et al: Heterozygous TGFBR2 mutations in Marfan syndrome. Nat Genet 2004, 36 (8): 855-860.

[6] Sakai H, Visser R, Ikegawa S, Ito E, Numabe H, Watanabe Y, Mikami H, Kondoh T, Kitoh H, Sugiyama R et al: Comprehensive genetic analysis of relevant four genes in 49 patients with Marfan syndrome or Marfan-related phenotypes. Am J Med Genet A 2006, 140 (16): 1719-1725.

[7] Singh KK, Rommel K, Mishra A, Karck M, Haverich A, Schmidtke J, Arslan-Kirchner M: TGFBR1 and TGFBR2 mutations in patients with features of Marfan syndrome and Loeys-Dietz syndrome. Hum Mutat 2006, 27 (8): 770-777.

[8] Loeys BL, Dietz HC, Braverman AC, Callewaert BL, De Backer J, Devereux RB, Hilhorst-Hofstee Y, Jondeau G, Faivre L, Milewicz DM et al: The revised Ghent nosology for the Marfan syndrome. J Med Genet 2010, 47 (7): 476-485.

[9] Pepe G, Giusti B, Sticchi E, Abbate R, Gensini GF, Nistri S: Marfan syndrome: current perspectives. Appl Clin Genet 2016, 9: 55-65.

[10] Dean JC: Marfan syndrome: clinical diagnosis and management. Eur J Hum Genet 2007, 15 (7): 724-733.

[11] Jones KB, Erkula G, Sponseller PD, Dormans JP: Spine deformity correction in Marfan syndrome. Spine (Phila Pa 1976) 2002, 27 (18): 2003-2012.

[12] Kurucan E, Bernstein DN, Ying M, Li Y, Menga EN, Sponseller PD, Mesfin A: Trends in spinal deformity surgery in Marfan syndrome. Spine J 2019, 19 (12): 1934-1940.

[13] Guala A, Teixido-Tura G, Rodriguez-Palomares J, Ruiz-Munoz A, Dux-Santoy L, Villalva N, Granato C, Galian L, Gutierrez L, Gonzalez-Alujas $\mathrm{T}$ et al: Proximal aorta longitudinal strain predicts aortic root dilation rate and aortic events in Marfan syndrome. Eur Heart J 2019, 40 (25): 2047-2055.

[14] Esfandiari H, Ansari S, Mohammad-Rabei H, Mets MB: Management Strategies of Ocular Abnormalities in Patients with Marfan Syndrome: Current Perspective. J Ophthalmic Vis Res 2019, 14 (1): 71-77.

[15] von Kodolitsch Y, De Backer J, Schuler H, Bannas P, Behzadi C, Bernhardt AM, Hillebrand M, Fuisting B, Sheikhzadeh S, Rybczynski $\mathrm{M}$ et al: Perspectives on the revised Ghent criteria for the diagnosis of Marfan syndrome. Appl Clin Genet 2015, 8: 137-155.

[16] Child AH: Non-cardiac manifestations of Marfan syndrome. Ann Cardiothorac Surg 2017, 6 (6): 599-609. 
[17] Asher MA, Burton DC: Adolescent idiopathic scoliosis: natural history and long term treatment effects. Scoliosis 2006, 1 (1): 2.

[18] Diarbakerli E, Grauers A, Danielsson A, Gerdhem P: Health-Related Quality of Life in Adulthood in Untreated and Treated Individuals with Adolescent or Juvenile Idiopathic Scoliosis. J Bone Joint Surg Am 2018, 100 (10): 811-817.

[19] Pehrsson K, Danielsson A, Nachemson A: Pulmonary function in adolescent idiopathic scoliosis: a 25 year follow up after surgery or start of brace treatment. Thorax 2001, 56 (5): 388-393.

[20] Yaszay B, Bastrom TP, Bartley CE, Parent S, Newton PO: The effects of the three-dimensional deformity of adolescent idiopathic scoliosis on pulmonary function. Eur Spine J 2017, 26 (6): 1658-1664. 